\title{
Reviewer Acknowledgements for Global Journal of Health Science, Vol. 6, No. 6
}

Global Journal of Health Science wishes to acknowledge the following individuals for their assistance with peer review of manuscripts for this issue. Their help and contributions in maintaining the quality of the journal is greatly appreciated.

Global Journal of Health Science is recruiting reviewers for the journal. If you are interested in becoming a reviewer, we welcome you to join us. Please find the application form and details at http://www.ccsenet.org/reviewer and e-mail the completed application form to gjhs@ccsenet.org.

\section{Reviewers for Volume 6, Number 6}

Alexander Domnich, University of Genoa, Italy

Ama Pokuaa Fenny, Aarhus University, Denmark

Ammar Eltayeb Ali Hassan, University of Tromsø, Norway

Anjan Ghosh, London \& KSS Deanery UK Faculty of Public Health, United Kingdom

Brian Cook, California State University Monterey Bay, United States

Caroline Elizabeth Bulsara, University of Western Australia, Australia

Christos Lionis, University of Crete, Greece

Darampal Dambhare, Mahatma Gandhi Institute of Medical Sciences, India

Domitila Augusta Huber, Federal University of Santa Catarina, Brazil

Farahnaz Amini, UCSI University, Malaysia

Fernanda Barbosa Lima-Christian, Federal University of Santa Catarina, Brazil

Francisco Rodenas Rigla, University of Valencia, Spain

Gabriele Messina, University of Siena, Italy

Jacob D. Christenson, Mount Mercy University, United States

Jason Tsai, University of Lincoln, United Kingdom

Jules Clement Assob, University of Buea, Cameroon

Kartheek R Balapala, University Tunku Abdul Rahman, Malaysia

Kim Solez, University of Alberta, Canada

Kimberley Geissler, Boston University School of Management, United States

Kingly Wangdi, Australian National University, Australia

Le Thi Thanh Xuan, Hanoi Medical University, Viet Nam

Loray Daws, British Columbia Masterson Institute, Canada

Maria Malliarou, echnological Institution of Larisa Nursing Department, Greece

Meng Zhao, Texas A\&M University at Corpus Christi, United States

Montarat Thavorncharoensap, HITAP, Ministry of Public Health, Thailand

Myo Nyein Aung, Juntendo University, Japan; Boromrajonani College of Nursing, Thailand

Nazisa Hejazi, National University, Malaysia

Pavlos Sarafis, Technological Educational Institute Lamia, Faculty of Nursing, Greece

Polly Yeung, Massey University, New Zealand

Raywat Deonandan, University of Ottawa, Canada

Samira Schultz Mansur, Federal University of Santa Catarina, Brazil

Shilpee Singh, University of Pennsylvania, United States

Srikrishna Sulgodu Ramachandra, Public Health Foundation of India, India

Tarek Tawfik Amin, King Faisal University, Saudi Arabia

Trisha Dunning, Deakin University and Barwon Health, Australia

Yevgeniy Samyshkin, IMS Health, United Kingdom

Zada Pajalic, School of Health and Society, Kristianstad University, Sweden 